\title{
Expression of Worst Case Using Multivariate Analysis in MOSFET Model Parameters
}

\author{
Takeshi Yasuda, Hiroshi Kawashima, Satoshi Hori, Member, IEEE, \\ Motoaki Tanizawa, Masao Yamawaki, and Sotoju Asai
}

\begin{abstract}
Device and circuit performance such as drain current and delay time varies stochastically due to uncontrollable factors in the fabrication processes. In this paper, a new method that represents the variation of the performance as worst case parameters in a MOSFET model is proposed. The variation of the performance can be expressed as a linear combination of several process-related parameters of the MOSFET model. Because of this fact, the worst case of parameters which corresponds to the worst case of performance can be directly and uniformly determined. Therefore, the calculation time of worst case parameters can be reduced by this method. The worst case parameter sets calculated by this method enable designers to estimate circuit performance variations accurately and easily. The capability of this method is verified in the variation analysis of drain current.
\end{abstract}

\section{INTRODUCTION}

D EVICE performance varies stochastically due to uncontrollable factors in the fabrication processes. Since miniaturization of semiconductor devices makes the performance more sensitive to process variation, more accurate and simple evaluation for the variation of performance is required in the early stages of device development.

Conventional methods which estimate the variation of performance using MOSFET model parameters of circuit simulation have been proposed. Table I compares the conventional methods. The principal component-based method [1]-[3] states that all the parameters of circuit simulation have to be extracted from capacitance-voltage $(I-V)$ curves. By using principal component analysis [4], all extracted parameters are transformed into principal components, and then all parameters are expressed as a linear combination of the parameters which have high correlation to the dominant principle components. After that, the worst corners of the parameters are determined and circuit simulation is utilized to find a set of parameters which corresponds to the worst case. Unfortunately, this conventional method is not computationally efficient, since the time required for selection of the worst case parameter sets increases as the square of the number of the dominant principle components.

The gradient method [5] states that, in the same way as the factor analytic technique, all parameters are extracted. These parameters are clustered into groups and then circuit

Manuscript received November 6, 1997; revised June 30, 1998

T. Yasuda, H. Kawashima, M. Tanizawa, M. Yamawaki, and S. Asai are with Mitsubishi Electric Corporation, ULSI Laboratory, Hyogo 664, Japan (e-mail: tayasuda@1si.melco.co.jp).

$\mathrm{S}$. Hori is with Mitsubishi Electric Corporation Manufacturing Engineering Center, Hyogo 661, Japan.

Publisher Item Identifier S 0894-6507(98)08366-3. simulation is utilized to calculate the sensitivity of each group of parameters to delay time of the circuit. After that, the variance of delay time is calculated from the sensitivity and the variance of the parameters. Unfortunately, this conventional method can only predict variance of delay time of the circuit and hence it can not predict worst case parameters.

In this paper, a new method is proposed for the calculation of parameters which represent the worst case of performance. Statistical analysis based on measurement data is described. The method is that device performance is measured and several parameters of circuit simulation are extracted. Performance variations can be expressed as a linear combination of several MOSFET model parameters using principal component regression analysis. Using this scheme, the worst case parameter is defined as the case in which the density function of distribution has the maximum value under given constant condition of performance. The worst case parameters calculated by this method allow designers to estimate the variation of device performance accurately and easily.

The outline of the method is described in Section II along with the calculation procedure. In Section III, the analysis of measurement data using various statistical approaches and the relationships between MOSFET model parameters and performance will be detailed and then the equation for calculating the worst case parameter is developed. Verification of the worst case parameter which corresponds to the worst value of performance is discussed in Section IV. Section V contains a summary and conclusion.

\section{OUTLINE OF THE METHOD}

The procedure of the new method is shown in Fig. 1. First, the drain current is measured from a large number of dies using the test element group (TEG), or predicted by the conventional method [5]. And then several parameters of circuit simulation are extracted from the dies using transistor TEG. Second, after the data points including outliers are eliminated from the data sets, the parameters are fitted to a suitable distribution. Third, a typical die with nominal performance is selected and all parameters of circuit simulation are extracted from it. Fourth, the parameters which represent the variation of performance are selected by alternated analysis. Fifth, we use multiple regression analysis to verify whether or not the variation of performance can be expressed as a linear combination of selected parameters. Sixth, the determination technique of worst case parameters is applied using statistical data of performance and the parameters. The parameter values 
TABLE I

Comparison Between Conventional and Proposed Methods

\begin{tabular}{|c|c|c|c|}
\hline Characteristic & $\begin{array}{l}\text { Principal component } \\
\text { based methoo }\end{array}$ & Gradient Method & Proposed method \\
\hline $\begin{array}{l}\text { Measurement } \\
\text { and } \\
\text { Extraction } \\
\end{array}$ & $\begin{array}{l}\text { I-V curves } \\
\text { All parameters }\end{array}$ & $\begin{array}{c}\text { I-V curves } \\
\text { All parameters }\end{array}$ & $\begin{array}{c}\text { Delay time of basic circuits } \\
\text { I-V curves (Linear region) } \\
\text { Several parameters } \\
\end{array}$ \\
\hline Technique & $\begin{array}{c}\text { Principal component analysis } \\
\text { Circuit simulation }\end{array}$ & $\begin{array}{l}\text { Cluster technique } \\
\text { Sensitivity analysis }\end{array}$ & $\begin{array}{l}\text { Principal component analysis } \\
\text { Multiple regression analysis }\end{array}$ \\
\hline Model & Model of circuit simulation & Model of circuit simulation & $\begin{array}{c}\text { Linear model } \\
\text { Distribution of parameter }\end{array}$ \\
\hline $\begin{array}{l}\text { Determination } \\
\text { technique of } \\
\text { worst case } \\
\text { parameter }\end{array}$ & Worst case corner & Calculation of variance & Linear constraint \\
\hline
\end{tabular}

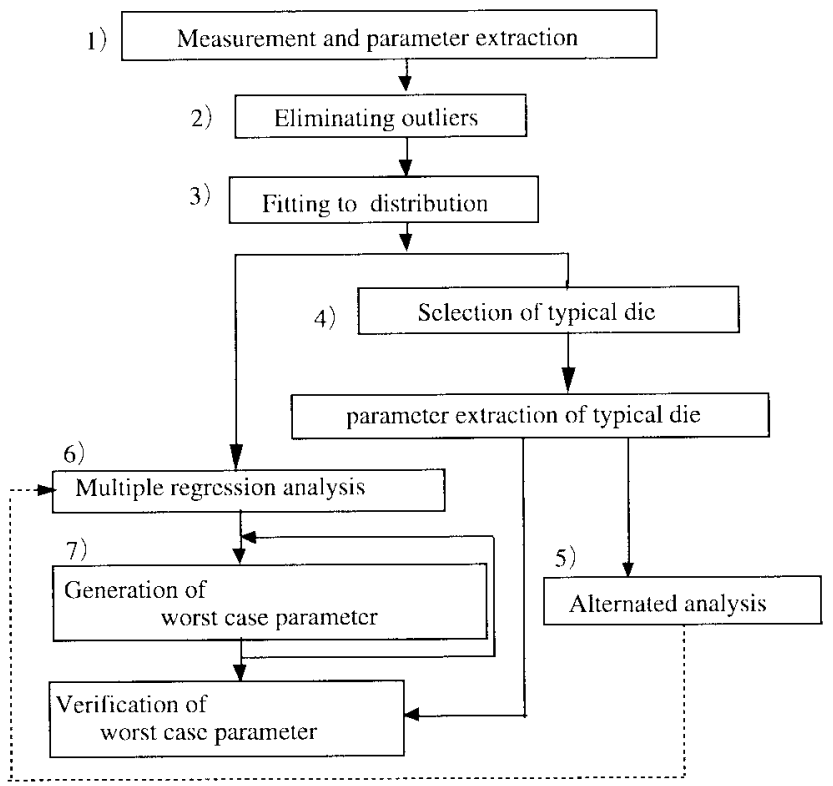

Fig. 1. Procedure of proposed method.

which correspond to worst case are determined. Finally, some of the typical parameters which represent the variation of performance are replaced by the worst case parameters and then the parameter sets can be verified by circuit simulations. The performance of various circuits can be analyzed by circuit simulations with this parameter set.

\section{Details OF THE Method}

Details of this method are as follows:

1) Measurement of Performance and Parameter Extraction: In the early stage of device development, transistor-TEG can be measured. Drain currents $\left(I_{d_{\max }}\right)$ are measured from a large number of dies using the transistor-TEG. The parameters, which are extracted using the transistor-TEG, are effective channel length $\left(L_{\text {eff }}\right)$, effective channel width ( $\left.W_{\text {eff }}\right)$, source/drain sheet resistance $(R S H)$, gate-oxide thickness $\left(T_{o x}\right)$ and threshold voltage $\left(V_{t h}\right)$. We selected these parameters from the viewpoint of their physical relationship to the device. Furthermore, the variation of performance can be represented by these parameters, as mentioned later. Therefore, even though the BSIM model has more parameters it is only necessary to extract these parameters in order to obtain various statistical information.

2) Eliminating Outliers: The data including outliers in a die are eliminated by the following two procedures. The first procedure is that the data which contain outliers are transformed into independent variables using principle component analysis, and then outliers are eliminated until the transformed parameters are uncorrelated. If there are only a few data points, however, a second procedure can be used, in which outliers are eliminated using histogram and correlation diagrams. As a result, a few data beyond 4 sigma of distribution were eliminated in this analysis.

3) Fitting to Suitable Distribution: The parameters and performances are fitted to a suitable distribution. The performance is fitted to a density function of normal distribution and the parameters are fitted to a density function of multivariate normal distribution as follows:

$$
\begin{aligned}
f(x)= & \exp \left\{-\left(x-\mu_{x}\right)^{T} \sum_{x}^{-1}\left(x-\mu_{x}\right) / 2\right\} \\
& /\left\{(2 \pi)^{n}\left|\sum_{x}\right|\right\}^{1 / 2} \cdots
\end{aligned}
$$

where $x$ is a parameter set, $\mu_{x}$ is the mean of each parameter, $n$ is a number of parameter, and $\sum_{x}$ is covariance matrix of $x$.

It is assumed that stochastic variables, which are a convolution of several uncontrollable factors, approximately follow a normal distribution. Fig. 2 shows examples of the fitting result. It can be seen that this assumption of performance and parameters is in good approximation. An example of correlation diagrams of parameters is shown in Fig. 3. Some of the parameters are strongly correlated with each other. Therefore, all the parameters mentioned above are fitted to a multivariate normal distribution to take into account the correlation between parameters.

4) Selection of Typical Die and Nominal Parameter Extraction: First, the dies whose performances are nearly mean in the distribution are selected. And then the die whose parameters mentioned above have the maximum density of 


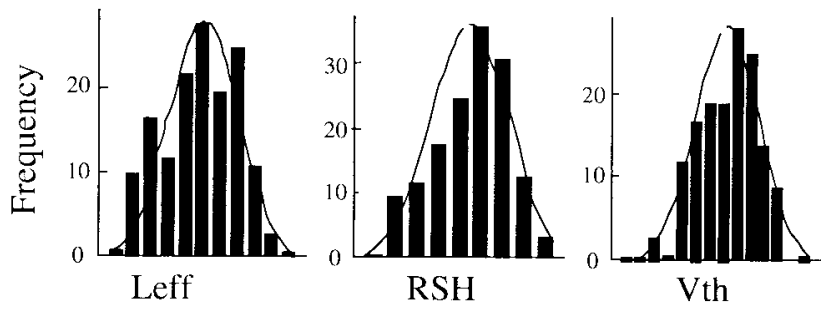

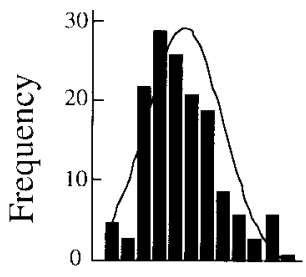

CJ

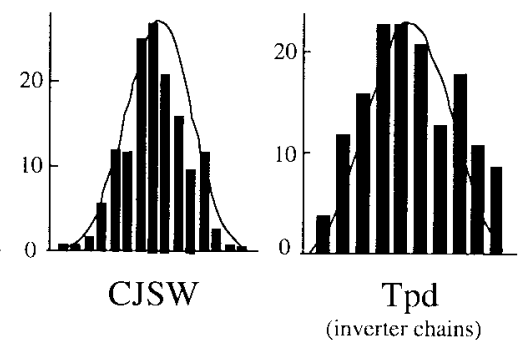

(inverter chains)
Fig. 2. Approximate results of fitting. Number of data is 157 in eight wafers.

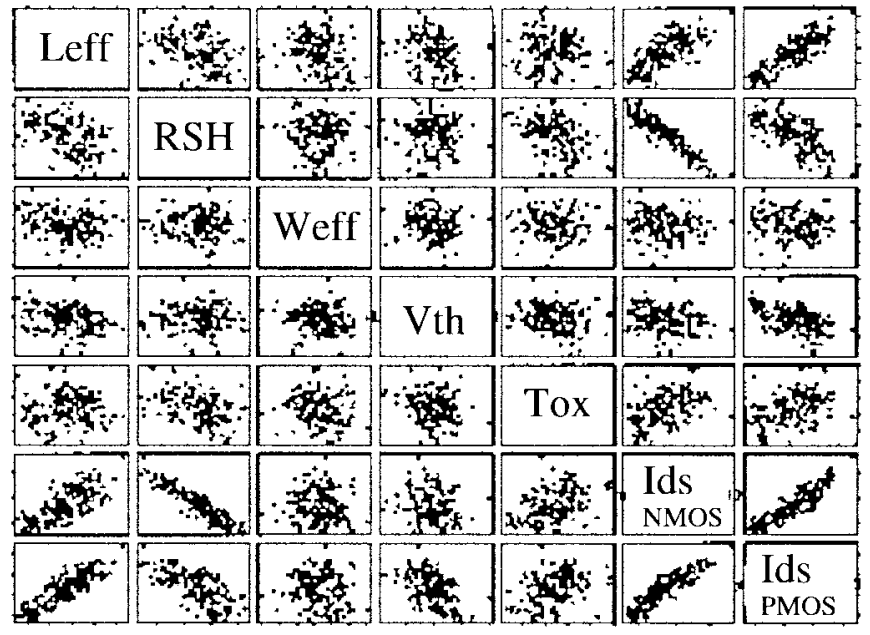

Fig. 3. Example of correlation diagrams of parameters. Vertical and horizontal axis in each plot correspond to parameter extended vertically and horizontally.

multivariate normal distribution is determined as the typical die of the selection. A set of nominal parameters of circuit simulation is extracted from the typical die. Nominal parameters are indispensable since a set of worst case parameter consists of nominal values and several calculated worst case parameters.

5) Parameter Selection Using the Alternated Analysis: It is desirable to represent the variation of performance by as few parameters as possible. Therefore, we have first selected several process-related parameters mentioned above which are directly concerned with the performance variation, and then we fixed the other parameters at constant values of the typical die.

Performances such as Idmax can be represented by only a few MOSFET model parameters of circuit simulation which make a large contribution to the variation of performance. To verify that the selected parameters can represent the performance variation, alternated analysis has been utilized. Details of the alternated analysis are as follows: some combination of parameters extracted from a measurement are selected and

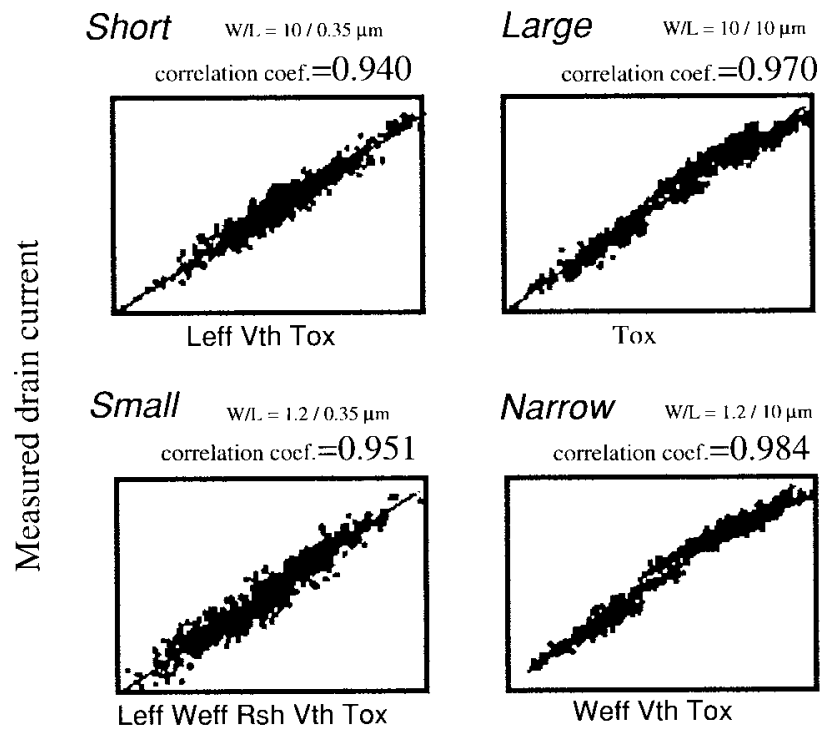

Simulated drain current

Fig. 4. Result of alternated analysis: vertical axis is measured drain current; horizontal axis is simulated drain current using extracted parameters as shown in each figure.

substituted into the typical parameter set. $I_{d_{\max }}$ is calculated by circuit simulation using this parameter set. This trial of analysis is repeated using data files of circuit simulation which are made from a combination of data for each parameter. Calculated $I_{d_{\max }}$ is compared with the measured $I_{d_{\max }}$ each other extended for whole measurement. Parameters which have a high correlation coefficient between simulated results and measurements are selected by alternation of parameter combination, though the parameters should be as few as possible. The results of the alternated analysis are shown in Fig. 4. As shown in this figure, only $T_{o x}$ is necessary to approximate variation in the case of large. In the case of short, three parameters of $L_{\mathrm{eff}}, V$ th, and $T_{o x}$ are necessary for approximation. Correlation coefficients $R$ between measured $I_{d_{\max }}$ vector and simulated $I_{d_{\max }}$ vector are larger than 0.94 for all four types of transistor. Therefore, the variation of $I_{d_{\max }}$ can be expressed as a function of at least $L_{\mathrm{eff}}, W_{\mathrm{eff}}, R S H$, $T_{o x}$, and $V_{t h}$ as a result of the alternated analysis.

6) The Identification of Contribution of Parameters Using Multiple Regression Analysis: From the result of the alternated analysis, the variation of performance can be represented as a function of the parameters mentioned above. Further, using Taylor series expansion for the function of the performance, the performance, for example $I_{d_{\max }}$, can be expressed as a linear combination of process-related parameters. At this point higher order terms such as second derivatives of each parameter are negligible compared to the first derivatives. The performance can be expressed as a linear combination of parameters

$$
p=\beta_{x}^{T}+\beta_{0}+e
$$

where $p$ is performance value or $k$ performances vector $\left(p_{1}, p_{2}, \cdots, p_{k}\right)^{T}, \quad x$ is $n$ parameters vector $\left(x_{1}, x_{2}, \cdots, x_{n}\right)^{T}, \beta$ is the regression coefficient vector or matrix, $\beta_{0}$ is a constant or vector of regression coefficient, 


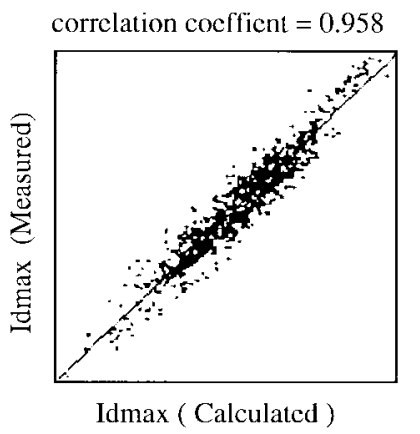

Fig. 5. Result of multiple regression analysis. Vertical axis is measured drain current; horizontal axis is calculated drain current by linear combination of extracted parameters using multiple regression analysis.

$T$ is the symbol of the transposition and $e$ is a vector of residual. For example, the variance of $I_{d_{\max }}$ is represented as a linear combination of $L_{\mathrm{eff}}, R S H$, and $V$ th,

$$
I_{d_{\max }}=\beta_{1} L_{\mathrm{eff}}+\beta_{2} R S H+\beta_{3} V \mathrm{th}+\beta_{0} .
$$

The result of multiple regression analysis is shown in Fig. 5. The correlation coefficient between measurement $I_{d_{\max }}$ and calculated $I_{d_{\max }}$ by linear combination of extracted parameters is 0.958 .

7) Determination Technique of Worst Case MOSFET Model Parameter: Parameters $x$ are transformed into principal components which are independent of each other

$$
z=A_{x}^{T}
$$

where $z$ is $n$ principal components vector $\left(z_{1}, z_{2}, \cdots, z_{n}\right)^{T}$ and $A$ is the characteristic matrix. It is necessary to transform the selected parameters into principal components, so that the multiple regression coefficient of principal components to performance can be calculated accurately and the correlation between parameters can be taken into account. Using this scheme, the variation of performance can now be expressed as a linear combination of several principal components by multiple regression analysis. Now, performance $p$ can be expressed as a linear combination of $m(m \leq n)$ principal components vector $\left(z_{1}, z_{2}, \cdots, z_{m}\right)^{T}$

$$
p=b^{T} z+b_{0}+e,
$$

where $b$ is a regression coefficient vector or matrix and $b_{0}$ is a constant or vector of regression coefficient. If residual $e$ can be neglected in (4) and $p$ is a constant value which corresponds to the worst case value of performance, the relationship in the equation can be expressed as

$$
b^{T} z=p-b_{0} .
$$

The worst case parameter $x_{w}$ is that $z$ satisfies (5) and the density of distribution has the maximum value. The worst case parameter $x_{w}$ can be calculated from (see Appendix I)

$$
x_{w}=\mu_{x}+\left(\sum_{x} B\right)\left(B^{T} \sum_{x} B\right)^{-1}\left(p-\mu_{p}\right)
$$

where $\mu_{x}$ is the mean of the parameter vector, $\mu_{p}$ is the mean of performance $b^{T} \mu_{z}+b_{0}$ or the performance of a typical die,

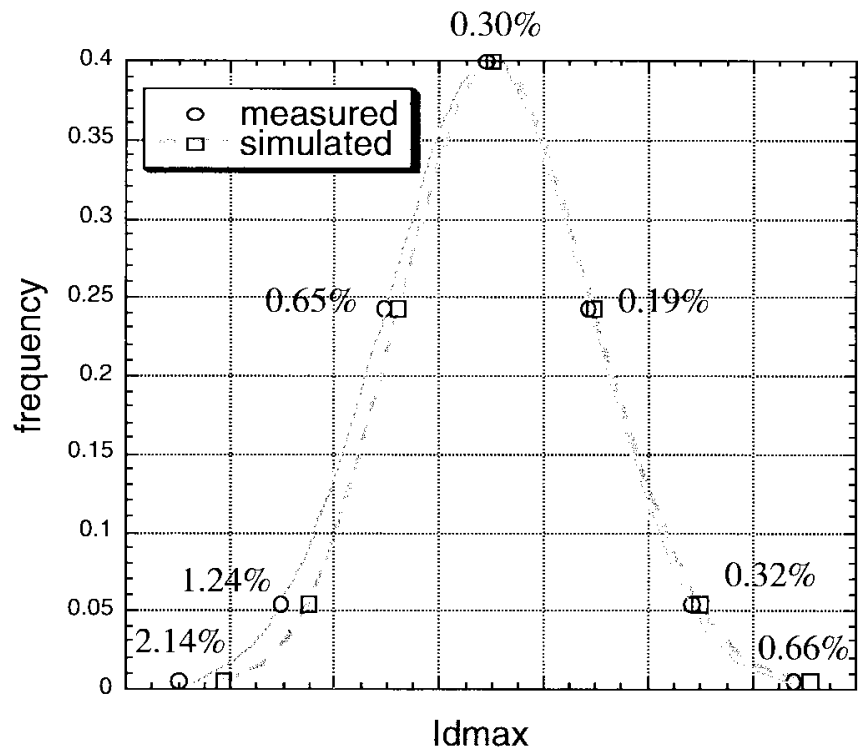

Fig. 6. Comparison of distribution between measured drain current and simulated one. Numeric in figure is error between measured and calculated drain current. Total data point is 816 in 16 wafers from eight lots.

$B$ is $A b$ or the sensitivity of the parameters to performances $\left[\partial p_{i} / \partial x_{j}\right](i=1,2, \cdots, k, j=1,2, \cdots, n)$. Parameter $x_{w}$ for circuit simulation can be uniformly calculated by substituting the performance $p$ which corresponds to the worst value of performance into (6). And then a set of worst case parameter consists of nominal values and calculated worst case parameters $x_{w}$. For example, the worst case parameter set in a MOSFET model is described as $\left(C_{1}, C_{2}, \cdots, L_{\mathrm{eff}_{w}}, R S H_{w}\right.$, $\left.V_{t h_{w}}, T_{o x_{m}}, \cdots, C_{n}\right)$, where $C_{1}, C_{2}$, and $C_{n}$ are extracted parameters, $T_{o x_{m}}$ is process related extracted parameters in the nominal chip. $L_{\mathrm{eff}_{w}}, R S H_{w}$, and $V_{t h_{w}}$ are calculated worst case parameters using (6). Moreover, this method includes the gradient method as shown in Appendix II.

\section{VERIFICATION}

We have applied this method to the variation analysis of $I_{d_{\max }}$ in order to verify its accuracy. Worst case parameters are calculated by the following procedure. The calculated $I_{d_{\max }}$ is substituted into (6) as the performance and several MOSFET model parameters such as $L_{\mathrm{eff}}, R S H$, and $V_{t h}$ are calculated.

Furthermore, the resulting values are the worst case parameters which most often appear in a given constant worst case performance value. The result shows the realistic worst case stochastically. In particular, this method also defined the worst case parameters being satisfied with (5).

A comparison between the measured $I_{d_{\max }}$ and simulated one using parameters calculated by this method is shown in Fig. 6. The error between the measured drain current and the drain current simulated using the resulting values is lower than $2.14 \%$.

\section{CONCLUSION}

A new method that represents the variation of performance by worst case parameters in a MOSFET model has been developed. A limited number of parameters were selected to 
represent the variation of performance from the viewpoint of their physical relationship to the device. Then, it was indicated by using alternated analysis that the performance variation can indeed be represented by the selected process-related parameters. Furthermore, the variation of performance can be expressed as a linear combination of selected process-related parameters by principal component regression analysis. Using this scheme, the worst case parameter has been defined as the case in which, given worst-case performance, the density of distribution has the maximum value. Therefore, we can easily estimate the variation of performance with several processrelated parameters. The error between the measured drain current and the drain current simulated using the resulting values is lower than $2.14 \%$.

The merits of this method are summarized as below:

1) The variations of performance can be expressed as several MOSFET model parameters by using principal component regression analysis.

2) The time which is required for calculation of worst case parameters is constant, since worst case MOSFET model parameters are directly and uniformly determined from the performance value.

3) The worst case parameters determined by this method are closer to reality stochastically than the conventional method using worst case corner.

4) The worst case parameters calculated by this method enable designers to estimate performance variations accurately and easily.

\section{APPENDIX I}

By using the method of undetermined multipliers, the worst case parameter $x_{w}$ is derived. Lagrangian function is

$$
L(x, \lambda)=\log (f(x))-\left(x^{T} B+B_{0}^{T}-p^{T}\right) \lambda
$$

where $\lambda$ undetermined multipliers vector.

$L$ is differentiated partially by each parameter and undetermined multipliers and then the following equation is obtained using (1),

$$
\begin{aligned}
\partial L(x, \lambda) / \partial x & =-\sum_{x}^{-1}\left(x-\mu_{x}\right)-B \lambda=0 \\
(\partial L(x, \lambda) / \partial \lambda)^{T} & =B^{T} x+B_{0}-p=0 .
\end{aligned}
$$

The following equation is obtained from (A2):

$$
x=\mu_{x}-\sum_{x} B \lambda .
$$

By substituting (A4) into (A3),

$$
\lambda=-\left(B^{T} \sum_{x} B\right)^{-1}\left\{p-\left(B^{T} \mu_{x}+B_{0}\right)\right\} .
$$

The worst case parameter $x_{w}$ is obtained by substituting (A5) into (A4):

$$
x_{w}=\mu_{x}+\left(\sum_{x} B\right)\left(B^{T} \sum_{x} B\right)^{-1}\left(p-\mu_{p}\right)
$$

where $\mu_{p}=B^{T} \mu_{x}+B_{0}$.

\section{APPENDIX II}

To derive into gradient method, the following equation is assumed first:

$$
p=\mu_{p}+B^{T}\left(x-\mu_{x}\right) .
$$

The following equation is obtained;

$$
\left(p-\mu_{p}\right)\left(p-\mu_{p}\right)^{T}=B^{T}\left(x-\mu_{x}\right)\left(x-\mu_{x}\right)^{T} B
$$

and

$$
\sum_{p}=B^{T} \sum_{x} B
$$

where $\sum_{p}$ is the covariance matrix of $p$. Therefore, this method includes the gradient method, as (A9) appears in (6). Moreover, if the number of performances $k$ is one, (6) states that the variation of parameters $\left(x-\mu_{x}\right)$ is the product of the term obtained by dividing the performance variation $\left(p-\mu_{p}\right)$ by the variance of performance $\left(B^{T} \sum_{x} B\right)$ and the contribution of the parameter to performance $\left(\sum_{x} B\right)$.

Only if the number of performances $k$ is equal to the number of parameters $n$, and $B$ is a regular matrix of $n$ degree, (6) becomes (A7). Additionally, if $\left(B^{T}\right)^{-1}$ can be assumed as $\left(B^{T}\right)^{+},(6)$ becomes the following equation;

$$
x_{w}=\mu_{x}+\left(B^{T}\right)^{+}\left(p-\mu_{p}\right)
$$

where + is the symbol of a generalized inverse [6].

Vice versa:

$$
\sum_{x}=\left(B^{T}\right)+\sum_{p} B^{+}
$$

\section{REFERENCES}

[1] F. Iravani, M. Habu, and E. Khalily, "Statistical modeling tools, methods and applications for integrated circuit manufacturability," in Proc. IEEE ICMTS'95, Mar. 1995, vol. 8, pp. 203-207.

[2] J. A. Power, B. Donnellan, A. Mathewson, and W. A. Lane, "Relating statistical MOSFET model parameter variabilities to IC manufacturing process fluctuations enabling realistic worst case design," IEEE Trans. Semiconduct. Manufact., vol. 7, pp. 306-318 Aug. 1994.

[3] J. A. Power, A. Mathewson, and W. A. Lane, "MOSFET statistical parameter extraction using multivariate statistics," in Proc. IEEE ICMTS'91, Mar. 1991, vol. 4, pp. 209-214.

[4] J. Edward Jackson, A User's Guide To Principal Components. New York: Wiley, 1991.

[5] M. Bolt, M. Rocchi, and J. Engel, "Realistic statistical worst-case simulations of VLSI circuit," IEEE Trans. Semiconuct. Manufact., vol. 4, pp. 193-198, Aug. 1991.

[6] C. Radhakrishna Rao, Linear Statistical Inference and Its Application. New York: Wiley, 1973

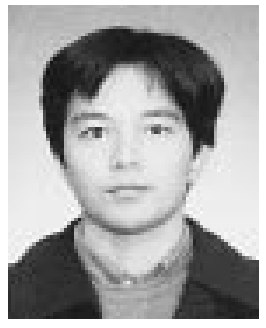

Takeshi Yasuda received the B.S. degree in industrial engineering from Kansai University, Osaka, Japan, in 1990 and the M.S. degree in mechanical engineering from Kansai University, Osaka, Japan, in 1992.

In 1992, he joined the ASIC Design Engineering Center, Mitsubishi Electric Corporation, Itami, Japan. In 1994, he joined the ULSI Laboratory, Mitsubishi Electric Corporation, Itami, Japan, where he is engaged in CMOS process development with special emphasis on the process estimation aspects. 


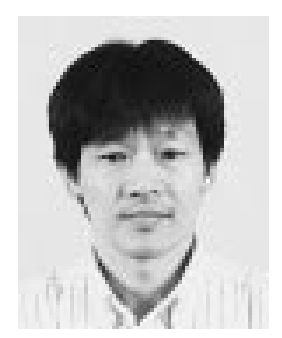

Hiroshi Kawashima received the B.E. degree in electrical engineering from the Science University of Tokyo, Tokyo, Japan, in 1983.

In 1983, he joined the Mitsubishi Electric Corporation ULSI Laboratory, Hyogo, Japan, where he has been engaged in the development of image sensors and CMOS process integration.

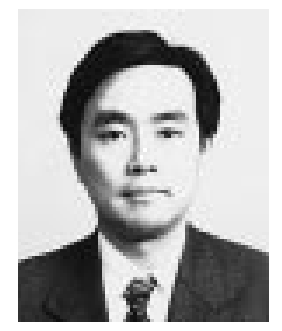

Satoshi Hori (S'86-M'87) received the B.E., M.E., and Doctor of Engineering degrees from the Tokyo Institute of Technology, Tokyo, Japan. He also received the M.S.E.E. degree from Purdue University, West Lafayette, IN.

$\mathrm{He}$ is currently working at the manufacturing system technology center, Mitsubishi Electric Corporation, Hyogo, Japan. His research interests include artificial intelligence, statistics in the fields of diagnosis, maintenance, quality control, and field service.

Dr. Hori is a Member of AAAI, Japanese Society of Artificial Intelligence, and IEIEC.

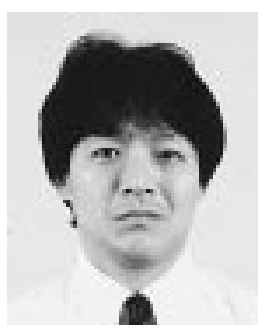

Motoaki Tanizawa was born in Osaka, Japan, in 1960. He received the B.S. and M.S. degrees in electronic engineering form Kyoto University, Kyoto, Japan, in 1983 and 1985, respectively.

From 1985 to 1988, he was with Sharp Corporation, Nara, Japan. In 1988, he joined Mitsubishi Electric Corporation, Hyogo, Japan, where he has been engaged in the development of semiconductor device modeling for circuit simulation.

Mr. Tanizawa is a member of the Japan Society of Applied Physics.

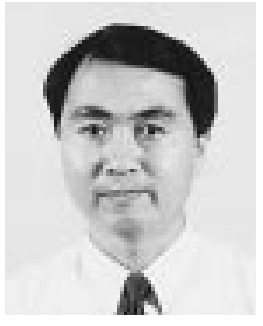

Physics.

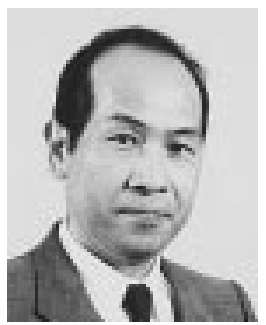

a Department Manager.
Masao Yamawaki received the B.S., M.S., and Doctor degrees in electronics engineering from Saitaima and Osaka University, Japan, in 1978, 1980, and 1997, respectively.

In 1980, he joined the LSI Laboratory, Mitsubishi Electric Corporation, Hyogo, Japan, where he has been engaged in the research and development of MOS memory devices and bipolar devices.

Dr. Yamawaki is a member of the Institute of Electronics, Information, and Communication Engineers of Japan and Japan Society of Applied

Sotoju Asai was born in Ishikawa, Japan in 1949 $\mathrm{He}$ received the B.S. degree in electronic engineering from Kanazawa University, Ishikawa, Japan in 1972 and the M.S. degree in electronic engineering from Tokyo Institute of Technology, Tokyo, Japan in 1974.

In 1974, he joined Central Research Laboratories, Mitsubishi Corporation. Since 1976, he has been engaged in development of MOS LSI process technology. He is currently working on CMOS logic process integration at ULSI Laboratory, where he is 